\title{
Short-Term Outcomes of Acute Low-Tone Sensorineural Hearing Loss According to Treatment Modality
}

\author{
Jinkyung Chang ${ }^{1}$, Gunhwee Yum², Ha-Young Im² $^{2}$, Jong Yoon Jung ${ }^{2}$, \\ Yoon Chan $\mathrm{Rah}^{2}$, and June $\mathrm{Choi}^{2}$ \\ ${ }^{1}$ The Graduate School, Korea University College of Medicine, Seoul, \\ ${ }^{2}$ Department of Otorhinolaryngology-Head and Neck Surgery, Korea University Ansan Hospital, Ansan, Korea
}

\begin{abstract}
Received February 4, 2016
Revised February 19, 2016

Accepted March 10, 2016
\end{abstract}

Background and Objectives: We compared improvements in hearing thresholds in acute low-tone sensorineural hearing loss (ALHL) patients after two different treatments: steroid alone and steroid and diuretic combined. We analyzed how the duration between the onset of symptoms and the initiation of treatment affected hearing loss improvement and investigated the relation between presence of vertigo in ALHL patients and ALHL progression to Ménière's disease (MD). Subjects and Methods: We retrospectively analyzed the medical records of $47 \mathrm{ALHL}$ patients aged 21 to 76 years. Patients received either orally administered steroid alone $(n=12)$ or steroid and diuretic combined $(n=35)$. We compared improvements in the two groups' hearing thresholds at three lower frequencies $(125,250$, and 500 $\mathrm{Hz}$ ) after participants had received one month of each respective treatment. Results: Our two treatments did not show any statistical difference in hearing loss improvement after one month. Forty percent of ALHL patients with vertigo developed MD, which was a significantly higher rate than the $12.5 \%$ of ALHL patients without vertigo who developed MD. The shorter duration between the onset of symptoms and the initiation of treatment significantly increased improvement in the sum of lower frequency hearing threshold after one month. Conclusions: The current study suggests that steroid and diuretic administered together and steroid alone similarly improve the hearing threshold in ALHL patients after one month. We concluded that patients should initiate ALHL treatment as soon as they experience symptoms. ALHL patients should also be notified of their higher risk of developing MD.

J Audiol Otol 2016;20(1):47-52

KEY WORDS: Acute low-tone sensorineural hearing loss · Steroid · Diuretic · Combination.

\section{Introduction}

Because hearing impairment is just one of the main symptoms of acute low-tone sensorineural hearing loss (ALHL) and because hearing often recovers without treatment, ALHL was previously neglected as an idiopathic sudden sensorineural hearing loss [1]. However, since 1982, ALHL has been regarded as a distinct disease entity from idiopathic sudden sensorineural hearing loss [2]. Several studies have investigated its pathophysiology and treatment. Diagnosis of ALHL is

This is an Open Access article distributed under the terms of the Creative Commons Attribution Non-Commercial License (http://creativecommons. org/licenses/by-nc/3.0/) which permits unrestricted non-commercial use, distribution, and reproduction in any medium, provided the original work is properly cited. based on subjective symptoms and objective hearing results. The specific hearing impairment of ALHL is mainly confined to the lower frequencies of 125,250 and $500 \mathrm{~Hz}$, with relatively normal hearing being maintained at higher frequencies of 2,4 , and $8 \mathrm{kHz}$ in the absence of vertigo and any structural damage $[3,4]$. The main symptoms of ALHL include lowpitched tinnitus, autophony, a feeling of fullness in the affected ear, and a weak sensation of dizziness [3]. ALHL patients often recover spontaneously but occasionally develop Ménière's disease (MD) and suffer from a recurrence of hearing loss in later years $[1,5]$.

Endolymphatic hydrops (EH) [5-9] and an autoimmune response [10] have been suggested as possible causes of ALHL. It has been reported that EH could induce MD [6,7,11], and 
EH patients with a positive finding on a glycerol test [8] showed an abnormally increased ratio of summating potential (SP) to action potential (AP) [9]. Considering that most ALHL patients also showed positive results on a glycerol test with increased SP on electrocochleography (ECoG) [5], EH might be an etiology for ALHL as well as MD.

Fuse, et al. [10] demonstrated Th1 lymphocyte predominance in the peripheral blood of both ALHL patients and MD patients. This imbalance between Th1 and Th2 lymphocytes implies the involvement of an immune response in the etiology of ALHL. According to previous studies, patients with ALHL have been administered diuretic, a treatment for $\mathrm{EH}$, and/or steroids to suppress the immune response.

Several studies have compared the efficacy of various treatments-steroid alone, diuretic alone, or steroid and diuretic combined-for ALHL patients [3,4,12-14]. Some studies have categorized and compared treatment outcomes [3,12], while others have concentrated on ALHL prognosis, such as hearing loss recurrence [13] or percentage of recovery [4], after different treatments. However, few studies have compared improvements in hearing thresholds at each lower frequency $(125,250$, and $500 \mathrm{~Hz})$ after the administration of different treatments.

The purpose of the present study was to investigate the clinical efficacy of steroid treatment and a combined steroid and diuretic treatment by evaluating improvements in hearing thresholds at lower frequencies $(125,250$, and $500 \mathrm{~Hz})$ in the affected ears after one month.

\section{Subjects and Methods}

\section{Data source and study population}

We retrospectively analyzed the medical records of 47 patients diagnosed with ALHL from 2009 to June 2014 at Korea University Ansan Hospital.

The diagnostic criteria for ALHL are not clearly defined, and different studies on ALHL appear to have established their own audiometric definition of ALHL [3,4,8,13,14]. For our study, we modified the audiometric definition of ALHL proposed by the Study Group for the Acute Profound Deafness Research Committee of the Ministry of Health, Labor and Welfare of Japan [3], as follows: 1) purely sensorineural hearing impairment with intact tympanic membranes; 2) no history of ocular, vestibular or brain injury; 3) $70 \mathrm{~dB}$ or greater sum of pure-tone audiogram hearing loss at three lower frequencies $(125,250$, and $500 \mathrm{~Hz})$ and $60 \mathrm{~dB}$ or less sum of hearing loss at three higher frequencies (2, 4, and $8 \mathrm{kHz}) ; 4$ ) no history of episodic vertigo or spontaneous nystagmus. Most patients complained of tinnitus, autophony, and a feeling of fullness in the affected ear.

The study population included 11 males and 36 females aged from 21 to 76 years, with a mean age of 43 years. Twelve patients were treated with steroid alone, while 35 patients received both steroid and diuretic. Steroid (methylprednisolone $64 \mathrm{mg}$ ) was administered orally for four days and then tapered during the following eight days (48 $\mathrm{mg}$ for two days, $32 \mathrm{mg}$ for two days, $24 \mathrm{mg}$ for one day, $16 \mathrm{mg}$ for one day, and $8 \mathrm{mg}$ for two days). Diuretic (hydrochlorothiazide $25 \mathrm{mg}$ ) was administered orally. The selection of treatment modalities was determined according to the preference of the clinical experience of doctors. In the current study, we analyzed the hearing threshold of the affected ear at frequencies of $125 \mathrm{~Hz}, 250 \mathrm{~Hz}, 500 \mathrm{~Hz}, 1 \mathrm{kHz}, 2 \mathrm{kHz}, 3 \mathrm{kHz}, 4 \mathrm{kHz}$, and $8 \mathrm{kHz}$ from audiograms of 47 patients, taken at the initial examination and also at the one-month follow-up visit. We then summed the hearing thresholds of the three lower frequencies after one month of treatment. We identified whether patients developed MD and investigated other possible prognostic factors, such as duration between the onset of symptoms and the initiation of treatment and presence of dizziness, diabetes mellitus (DM), or hypertension (HTN).

\section{Statistical analysis}

The clinical parameters of age, sex, DM, HTN, initial hearing thresholds, duration between the onset of symptoms and the initiation of treatment, treatment outcome after one month, and relationship between the presence of dizziness and the development of MD were analyzed for patients with ALHL. Mann-Whitney U test was used to evaluate whether the duration between the onset of symptoms and the initiation of treatment influenced the treatment. Independent $t$-test was used to assess whether age or initial hearing levels influenced the type of treatment patients received and also to compare improvements in hearing thresholds after one month for patients receiving one of two different treatments. Paired t-test was used to evaluate whether each treatment significantly improved the initial hearing thresholds after one month. We used the $\chi^{2}$ test to determine whether age, sex, or presence of DM or HTN affected the treatment received and to identify the relation between presence of dizziness and development of MD. Finally, Spearman's correlation test was used to assess whether the duration between the onset of symptoms and the initiation of treatment influenced hearing improvement. For all statistical tests, a $p$-value less than 0.05 was considered significant. Statistical analysis was performed with Statistical Package for the Social Sciences version 18.0 (IBM, Armonk, NY, USA). This study was reviewed and approved by the Korea University Ansan Hospital Institutional Review Board. 


\section{Results}

\section{Demographic data}

Of our 47 total patients, 12 were administered steroid alone and 35 received steroid and diuretic together. Eleven males and 36 females with a mean age of 43 years initiated treatment an average of 11.5 days after their first experience of ALHL symptoms. Four patients suffered from DM, and six patients had HTN. ALHL patients in the steroid-alone treatment group had an average age of $48.2 \pm 17.1$ years, while those in the combination treatment group had an average age of $40.3 \pm 10.3$ years. Patient characteristics are summarized in Table 1 .

\section{Clinical data}

Three of 12 patients $(25 \%)$ in the steroid-alone treatment group suffered from DM, while one of 35 patients $(2.9 \%)$ in the combination treatment group reported DM. Thus, the steroid-alone treatment group had a significantly increased ratio of ALHL patients with DM due to of the small number of patients. However, cases were too small to be generalized. The mean hearing threshold of the three lower frequencies in the affected ears was $45.9 \mathrm{~dB}$ in male patients and $40.9 \mathrm{~dB}$ in female patients. The factors of sex, affected ear, elapsed time between the onset of symptoms and the initiation of treatment, presence of HTN, and initial hearing threshold at each lower frequency $(125,250$, and $500 \mathrm{~Hz})$ were not significantly different between the two treatment groups. Table 2 summarizes clinical data according to treatment group.

Age, sex, presence of HTN, initial hearing loss measured at each lower frequency, and the sum of lower frequency

Table 1. Demographic data

\begin{tabular}{lccc}
\hline & $\begin{array}{c}\text { Steroid } \\
\text { only }\end{array}$ & $\begin{array}{c}\text { Steroid and } \\
\text { diuretic combined }\end{array}$ & $\begin{array}{c}\mathrm{P} \text { - } \\
\text { value }\end{array}$ \\
\hline Number & 12 & 35 & 0.098 \\
Sex $(\mathrm{M}: \mathrm{F})$ & $5: 7$ & $6: 29$ & 0.113 \\
Age (years) (mean $\pm \mathrm{SD})$ & $48.2 \pm 17.1$ & $40.3 \pm 10.3$ & 0.009 \\
Side (R:L) & $7: 5$ & $16: 19$ & 0.092 \\
\hline
\end{tabular}

M: male, F: female, $\mathrm{R}$ : right, L: Left, SD: standard deviation hearing thresholds did not affect the type of treatment patients received.

\section{Presence of dizziness significantly increased chance of developing MD}

ALHL patients with dizziness at the initial examination had a significantly increased chance of developing MD (6/15 patients, $40 \%$ ) at a later date compared to patients without dizziness (4/32 patients, $12.5 \%)(p=0.037)$ (Fig.1). The treatment modality did not influence development of MD ( $p=$ 0.48). Three of 12 ALHL patients who received only steroid developed MD (25\%), while seven of 35 patients who were given both steroid and diuretic developed MD (20\%).

\section{Hearing improvements at lower frequencies after one month}

After one month of treatment with steroid alone, ALHL patients experienced significantly improved hearing thresholds

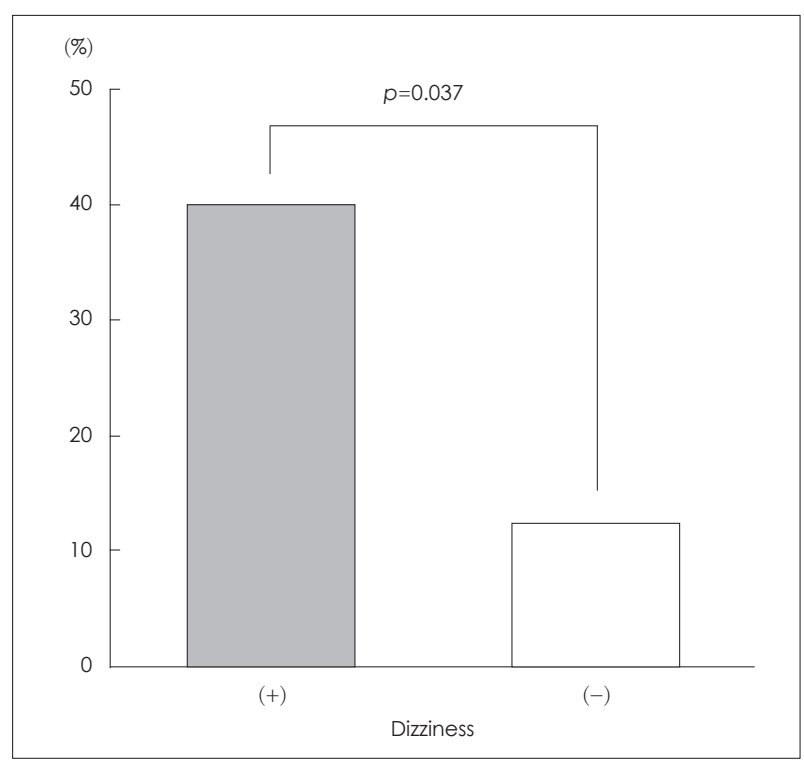

Fig. 1. Progression to Ménière's disease (MD). ALHL patients with dizziness had a significantly increased chance of developing MD (6/15 patients, $40 \%)$ compared to patients without dizziness (4/32 patients, $12.5 \%)(p=0.037)$. ALHL: acute low-tone sensorineural hearing loss.

Table 2. Clinical data

\begin{tabular}{lrrc}
\hline & Steroid only & Steroid and diuretic combined & $p$-value \\
\hline Duration from onset to initial visit (days) (mean \pm SD) & $16.5 \pm 18.8$ & $9.8 \pm 14.8$ & 0.150 \\
DM & $25.0 \%(3 / 12)$ & $2.9 \%(1 / 35)$ & 0.043 \\
HTN & $16.7 \%(2 / 12)$ & $11.4 \%(4 / 35)$ & 0.078 \\
Hearing level at $125 \mathrm{~Hz}(\mathrm{~dB})($ mean \pm SD) & $48.3 \pm 17.9$ & $41.6 \pm 14.6$ & 0.169 \\
Hearing level at $250 \mathrm{~Hz}(\mathrm{~dB})($ mean \pm SD) & $48.8 \pm 20.6$ & $43.0 \pm 14.5$ & 0.262 \\
Hearing level at $500 \mathrm{~Hz}(\mathrm{~dB})($ mean \pm SD) & $44.6 \pm 23.5$ & $37.6 \pm 15.5$ & 0.219 \\
Sum of hearing level at three lowest frequencies (dB) (mean $\pm S D)$ & $141.7 \pm 59.4$ & $122.1 \pm 43.0$ & 0.197 \\
\hline
\end{tabular}

DM: diabetes mellitus, HTN: hypertension, SD: standard deviation 
by $14.5 \mathrm{~dB}$ at $125 \mathrm{~Hz}(p=0.011), 15 \mathrm{~dB}$ at $250 \mathrm{~Hz}(p=0.011)$, and $14.6 \mathrm{~dB}$ at $500 \mathrm{~Hz}(p=0.011)$. Hearing improvements were statistically significant. After one month of steroid and diuretic treatment, the hearing thresholds improved by 14.4 $\mathrm{dB}$ at $125 \mathrm{~Hz}(p=0.009), 17.2 \mathrm{~dB}$ at $250 \mathrm{~Hz}(p=0.008)$, and $14.8 \mathrm{~dB}$ at $500 \mathrm{~Hz}(p=0.011)$, which are also statistically significant (Table 3).

\section{Differences between improvements in hearing thresholds between groups}

Patients administered only steroid experienced an improvement in hearing thresholds by an average of $14.6 \mathrm{~dB}$ at 125 $\mathrm{Hz}, 15.0 \mathrm{~dB}$ at $250 \mathrm{~Hz}$, and $14.6 \mathrm{~dB}$ at $500 \mathrm{~Hz}$ after one month, while those who received both steroid and diuretic together improved by an average of $14.4 \mathrm{~dB}$ at $125 \mathrm{~Hz}, 17.1 \mathrm{~dB}$ at $250 \mathrm{~Hz}$, and $14.8 \mathrm{~dB}$ at $500 \mathrm{~Hz}$ in the same time period. The differences between groups with regard to improvements in hearing thresholds after one month were not significant ( $p=0.48, p=0.32$, and $p=0.46$ at 125,250 , and $500 \mathrm{~Hz}$, respectively). Improvements in the sum of the hearing thresholds of three lower frequencies after one month were $44.2 \mathrm{~dB}$ in the steroid-only treatment group and $47.6 \mathrm{~dB}$ in the combination treatment group, which was not significant $(p=0.42)$ (Table 4$)$.

\section{Duration between the onset of symptoms and the} initiation of treatment influenced hearing improvement after one month

According to Spearman's correlation test, the amount of time that passed between the onset of symptoms and the initiation of treatment significantly influenced hearing improvements amongst the sum of hearing thresholds at the three lower frequencies after one month of treatment $(p=0.008)$. Patients with ALHL took an average of 11.5 days to initiate treatment. The Spearman's correlation coefficient was -0.384 .

\section{Discussion}

The current study investigated the clinical efficacy of two kinds of treatments for ALHL patients: a steroid-only treatment and a steroid-diuretic combination treatment. We compared the improvements in hearing thresholds at each lower frequency $(125,250$, and $500 \mathrm{~Hz})$ in affected ears between the two treatment groups after one month of treatment. Both the steroid alone and the combined steroid and diuretic significantly improved hearing threshold at each frequency after one month. The steroid-only treatment and the combination treatment enhanced hearing thresholds by a similar extent after one month, with no significant difference between the improvements.

These findings suggest that steroid alone might be sufficient as a short-term treatment for ALHL patients. Diuretics have been administered to patients with ALHL $[15,16]$ based upon the assumption that the etiology of ALHL involves EH and MD. ALHL patients often test positive on a glycerol test [8] and display an increased ratio of SP to AP on ECoG. A positive glycerol test and similar ECoG findings are also recognized in patients with EH and MD [5,9], suggesting EH as

Table 3. Hearing thresholds at each lower frequency for the steroid-only treatment group and the steroid and diuretic combined treatment group

\begin{tabular}{cccccc}
\hline & Frequency $(\mathrm{Hz})$ & $\begin{array}{c}\text { Initial hearing } \\
\text { threshold }(\mathrm{dB})\end{array}$ & $\begin{array}{c}\text { Hearing threshold } \\
\text { after one month }(\mathrm{dB})\end{array}$ & $\begin{array}{c}\text { Percentage reaching a normal } \\
\text { hearing threshold level (\%) }\end{array}$ & $\begin{array}{c}p- \\
\text { value }\end{array}$ \\
\hline Steroid-only treatment $(\mathrm{n}=12)$ & 125 & 48.3 & 33.8 & $25(3 / 12)$ & 0.011 \\
& 250 & 48.8 & 33.8 & $25(3 / 12)$ & 0.011 \\
Steroid and diuretic combined & 125 & 44.6 & 30.0 & $41.7(5 / 12)$ & 0.011 \\
treatment (n=35) & 250 & 42.6 & 26.7 & $42.9(15 / 35)$ & 0.009 \\
& 500 & 37.2 & 25.4 & $51.4(18 / 35)$ & 0.007 \\
& 500 & 22.4 & $60(21 / 35)$ & 0.011 \\
\hline
\end{tabular}

A normal hearing threshold at each decibel is assumed to be less than $25 \mathrm{~dB}$

Table 4. Treatment options and hearing loss improvements after one month

\begin{tabular}{|c|c|c|c|}
\hline Frequency $(\mathrm{Hz})$ & Treatment & $\begin{array}{c}\text { Average improvement in hearing } \\
\text { thresholds after one month }(\mathrm{dB})(\text { mean } \pm \mathrm{SD})\end{array}$ & $p$-value \\
\hline \multirow[t]{2}{*}{125} & Steroid only $(n=12)$ & $14.6 \pm 19.1$ & 0.480 \\
\hline & Steroid and diuretic combined $(n=36)$ & $14.4 \pm 14.7$ & \\
\hline \multirow[t]{2}{*}{250} & Steroid only $(n=12)$ & $15.0 \pm 19.5$ & 0.320 \\
\hline & Steroid and diuretic combined $(n=36)$ & $17.2 \pm 15.6$ & \\
\hline \multirow[t]{2}{*}{500} & Steroid only $(n=12)$ & $14.6 \pm 19.0$ & 0.460 \\
\hline & Steroid and diuretic combined $(n=36)$ & $14.8 \pm 15.6$ & \\
\hline Sum of hearing thresholds & Steroid only $(n=12)$ & $44.2 \pm 56.4$ & 0.420 \\
\hline at 125,250 , and $500 \mathrm{~Hz}$ & Steroid and Diuretic combined $(n=6)$ & $47.6 \pm 43.9$ & \\
\hline
\end{tabular}


an etiology of ALHL and ALHL as an early stage of MD. Thus, diuretics, which are known treatments for $\mathrm{EH}$ and $\mathrm{MD}$, began to be administered to patients with ALHL.

The clinical effects of diuretics in improving hearing thresholds in ALHL patients have been debated. Morita, et al. [3] reported improved hearing loss in $75 \%$ of their ALHL patients using a diuretic-only treatment, while Suzuki, et al. [4] observed that the administration of diuretic alone did not significantly improve hearing loss. However, Suzuki, et al. [4] evaluated the effects of a diuretic alone after one month, during which the diuretics might not have had enough time to exhibit their therapeutic effects. Nozawa, et al. [16] demonstrated that, when administered long-term, diuretic alone significantly improved hearing loss in patients with MD. They concluded that a diuretic should be prescribed to patients with MD for at least several months before its clinical efficacy is evaluated [16]. However, because only $75 \%$ of ALHL patients experienced hearing loss improvements from a diuretic-only treatment [3], another mechanism may have a role in ALHL in addition to EH. Our finding that a combined diuretic and steroid treatment improved hearing thresholds to a similar extent as a steroid-alone treatment is supportive of the long-term therapeutic effects of diuretics for ALHL treatment.

We discovered that treatment with steroid alone improved the hearing thresholds at lower frequencies in ALHL patients to a similar extent as the combination treatment. This result implied the involvement of an immune response in the etiology of ALHL. Based on his diagnostic studies and his patients' responsiveness to an immunosuppressant, such as cortisone, McCabe [17] was the first to recognize an autoimmune response in patients with sensorineural hearing loss. Reports on the immunologic state of ALHL patients by measuring concentrations of Th1 and Th2 lymphocytes in the peripheral blood of ALHL patients and an abnormal increase in concentration of Th1 compared to that of the control group soon followed [10]. It was suggested that patients with MD display significantly increased activity of natural killer cells [10]. The imbalance between Th1/Th2 lymphocytes and increased immunologic activity in ALHL and MD patients indicates that the etiologies of ALHL and MD involve an immune response in the endolymphatic sac and the inner ear. Fuse, et al. [10] concluded that ALHL and MD were different stages of the same immunological inner ear disease; ALHL is the early stage, and MD is the late stage. However, this increased immune response in ALHL and MD patients does not indicate a causal relationship between the immune response and ALHL or MD. Future research should investigate the causes of increased immune response in ALHL patients, the biochemical mediators and receptors involved, and the relationship be- tween $\mathrm{EH}$ and increased immune response in ALHL patients. The duration between symptom onset and treatment initiation significantly influenced hearing improvements made within one month of both a steroid treatment alone and a combination treatment. Patients with ALHL initiated treatment after an average of 11.5 days from their first experience of ALHL symptoms. A negative correlation existed between the amount of time patients waited before initiating treatments and the extent of hearing improvements made within one month. Therefore, when patients waited longer to initiate treatment, less hearing improvement was made after one month of treatment. Earlier studies also reported that the percentage recovery of hearing significantly increased when patients started their ALHL treatment within seven days of ALHL symptom appearance [4].

Some studies have also observed hearing loss recurrence [13] and percentage of recovery [4] after differing treatments. Fuse, et al. [12] categorized hearing loss improvements using an audiometric definition, reporting that many ALHL patients achieved complete recovery between 7 and 10 days after administration of steroid. Patients who did not achieve complete or partial recovery-about $20 \%$ of the tested ALHL patientstended to report a poor recovery in long-term follow-up [12]. Therefore, the short-term prognosis for hearing seems to be determined within one month after initiation of treatment. Regarding ALHL status and development of MD, we demonstrated that patients experiencing dizziness had a higher chance of developing MD (40\%) compared to patients not experiencing dizziness (12.5\%) (Fig. 1). Treatment modality, whether steroid only or steroid and diuretic together, did not influence the development of MD. The relationship between ALHL and MD can be understood on a continuum of EH [18]. Several studies have suggested ALHL as an early-stage of EH confined to the cochlea [5,17]. Because EH is restricted to the cochlea, patients in the initial state would not experience dizziness. As cochlear hydrops advances to the vestibular apparatus, patients with EH might develop dizziness with ALHL progression to MD $[5,18]$. From this perspective, ALHL without dizziness is considered to be an early stage of $\mathrm{EH}$, while $\mathrm{MD}$ is thought to be a late stage of $\mathrm{EH}$. However, some ALHL patients with dizziness do not develop MD, and others who do not report dizziness develop MD. Therefore, prognostic factors other than EH must be involved in ALHL patients who develop MD.

Some studies have investigated the prediction of progression to MD in ALHL patients without dizziness or in patients with idiopathic sudden hearing loss without vertigo. It was reported that $12.5 \%$ of patients with ALHL who displayed spontaneous nystagmus on initial electronystagmography de- 
veloped MD despite receiving treatment with steroid alone for eight days [13]. However, none of the ALHL and non-ALHL patients without spontaneous nystagmus developed MD [13].

In addition, the cochlear hydrops analysis masking procedure may detect changes in cochlear response in ALHL patients not experiencing dizziness and may predict whether a patient will develop MD [18].

In conclusion, the etiology of ALHL and its treatments are not yet clear. We compared improvements in hearing loss of ALHL patients treated with steroid alone or those who received both steroid and diuretic. The two treatments both significantly improved hearing loss in ALHL patients after one month. However, the combination treatment for ALHL had a greater tendency to improve hearing loss at lower frequencies than did the steroid-only treatment. In addition, patients should initiate ALHL treatment as soon as they experience ALHL symptoms in order to increase the efficacy of treatment. ALHL patients with vertigo should also be made aware of the possible development of MD.

\section{Conflicts of interest}

The authors have no financial conflicts of interest.

\section{REFERENCES}

1) Imamura $S$, Nozawa I, Imamura M, Murakami Y. Clinical observations on acute low-tone sensorineural hearing loss. Survey and analysis of 137 patients. Ann Otol Rhinol Laryngol 1997;106:746-50.

2) Abe $T$. Acute sensorineural hearing loss in low tone frequencies. Otolaryngol Tokyo 1982;54:385-92.

3) Morita S, Suzuki M, Iizuka K. A comparison of the short-term outcome in patients with acute low-tone sensorineural hearing loss. ORL J Otorhinolaryngol Relat Spec 2010;72:295-9.

4) Suzuki M, Otake R, Kashio A. Effect of corticosteroids or diuretics in low-tone sensorineural hearing loss. ORL J Otorhinolaryngol Relat Spec 2006;68:170-6.

5) Yamasoba T, Kikuchi S, Sugasawa M, Yagi M, Harada T. Acute low-tone sensorineural hearing loss without vertigo. Arch Otolaryngol Head Neck Surg 1994;120:532-5.

6) Aso S, Kimura H, Takeda S, Mizukoshi K, Watanabe Y. The intravenously administered glycerol test. Acta Otolaryngol Suppl 1993; 504:51-4.

7) Merchant SN, Adams JC, Nadol JB Jr. Pathophysiology of Meniere's syndrome: are symptoms caused by endolymphatic hydrops? Otol Neurotol 2005;26:74-81.

8) Nozawa I, Imamura S, Mizukoshi A, Honda H, Okamoto Y. Clinical study of acute low-tone sensorineural hearing loss: survey and analysis of glycerol test and orthostatic test. Ann Otol Rhinol Laryngol 2002;111:160-4.

9) Yamasoba T, Sugasawa M, Kikuchi S, Yagi M, Harada T. An electrocochleographic study of acute low-tone sensorineural hearing loss. Eur Arch Otorhinolaryngol 1993;250:418-22.

10) Fuse T, Hayashi T, Oota N, Fukase $S$, Asano $S$, Kato T, et al. Immunological responses in acute low-tone sensorineural hearing loss and Ménière's disease. Acta Otolaryngol 2003;123:26-31.

11) Foster CA, Breeze RE. Endolymphatic hydrops in Ménière's disease: cause, consequence, or epiphenomenon? Otol Neurotol 2013; 34:1210-4.

12) Fuse T, Aoyagi M, Funakubo T, Sakakibara A, Yoshida S. Shortterm outcome and prognosis of acute low-tone sensorineural hearing loss by administration of steroid. ORL J Otorhinolaryngol Relat Spec 2002;64:6-10.

13) Fushiki H, Junicho M, Kanazawa $Y$, Aso $S$, Watanabe Y. Prognosis of sudden low-tone loss other than acute low-tone sensorineural hearing loss. Acta Otolaryngol 2010;130:559-64.

14) Roh KJ, Lee EJ, Park AY, Choi BI, Son EJ. Long-term outcomes of acute low-tone hearing loss. J Audiol Otol 2015;19:74-8.

15) Kanda K, Watanabe Y, Shojaku H, Ito M, Mizukoshi K. Effects of isosorbide in patients with Menière's disease. Acta Otolaryngol Suppl 1993;504:79-81.

16) Nozawa I, Nakayama $H$, Hashimoto $K$, Imamura $S$, Hisamatu $K$, Murakami Y. Efficacy of long-term administration of isosorbide for Ménière's disease. ORL J Otorhinolaryngol Relat Spec 1995;57:13540.

17) McCabe BF. Autoimmune sensorineural hearing loss. Ann Otol Rhinol Laryngol 1979;88(5 Pt 1):585-9.

18) Hong SK, Nam SW, Lee HJ, Koo JW, Kim DH, Kim DR, et al. Clinical observation on acute low-frequency hearing loss without vertigo: the role of cochlear hydrops analysis masking procedure as initial prognostic parameter. Ear Hear 2013;34:229-35. 